\title{
Labyrinthe
}

18 | 2004 (2)

La Recherche dans tous ses éclats

\section{« Nouvelles perspectives de recherche sur la couronne d'Aragon au moyen âge "}

Journée d'études doctorales (Institució Milà i Fontanals-CSIC et Casa de Velázquez, coord. D. Duran i Duelt et S. Péquignot, Barcelone, 20 octobre 2003)

\section{Stéphane Péquignot}

\section{OpenEdition}

1 Journals

Édition électronique

URL : http://journals.openedition.org/labyrinthe/224

DOI : $10.4000 /$ labyrinthe.224

ISSN : 1950-6031

Éditeur

Hermann

Édition imprimée

Date de publication : 15 juillet 2004

Pagination : 85-87

Référence électronique

Stéphane Péquignot, « " Nouvelles perspectives de recherche sur la couronne d'Aragon au moyen

âge » », Labyrinthe [En ligne], 18 | 2004 (2), mis en ligne le 24 juin 2008, consulté le 19 avril 2019. URL: http://journals.openedition.org/labyrinthe/224 ; DOI : 10.4000/labyrinthe.224

Propriété intellectuelle 


\title{
«NOUVELLES PERSPECTIVES DE RECHERCHE SUR LA COURONNE D'ARAGON AU MOYEN ÂGE»
}

\author{
Journée d'études doctorales \\ (Institució Milà i Fontanals-CSIC et Casa de Velázquez, \\ coord. D. Duran i Duelt et S. Péquignot, Barcelone, \\ 20 octobre 2003)
}

\author{
Stéphane PÉQUIGNOT \\ stephanepequignot@yahoo.fr
}

Depuis plusieurs années déjà, les séminaires de doctorants et les journées d'études doctorales ont acquis leur place et démontré leur utilité dans les calendriers annuels des universités françaises. Ces deux pratiques demeurent néanmoins relativement rares en Espagne, où les travaux des doctorants en sciences sociales s'effectuent souvent dans un grand isolement que la parcimonie et la maigreur des bourses rendent plus amer encore. Les recherches menées sur la couronne d'Aragon au Moyen Âge (histoire, histoire de l'art, architecture, littérature) n'échappent pas à la règle. Aux problèmes institutionnels et financiers déjà évoqués s'ajoutent cependant ici les difficultés inhérentes à un objet particulièrement hétéroclite, puisque les territoires placés à un moment ou à un autre sous la domination de la dynastie aragonaise (comté de Catalogne, royaumes d'Aragon, de Valence, de Majorque, de Sicile, de Sardaigne et autres possessions en Méditerranée orientale) relèvent le plus souvent de traditions historiographiques nationales et disciplinaires multiples, encore trop étanches. Pour tenter de rompre avec le double isolement des thésards et des historiographies, un séminaire interdisciplinaire mensuel de doctorants a été fondé en 2002 au CSIC (Consejo superior de investigaciones científicas, l'équivalent espagnol du CNRS) à Barcelone. Son objet était triple : faciliter la circulation d'informations d'accès souvent difficile (bibliographies, archives, etc.), permettre la rencontre entre doctorants espagnols et étrangers de passage dans la capitale catalane et, surtout, créer un espace de dialogue critique et non 
hiérarchique dans lequel chacun pourrait exposer ses hypothèses de travail et les soumettre au débat. Le 20 octobre 2003, au CSIC, la présentation en catalan, en castillan et en français de ces «nouvelles perspectives de recherche sur la couronne d'Aragon au Moyen Âge» constituait l'aboutissement public de ce séminaire international.

Dans un cadre volontairement assez informel - les bureaux ont été descendus de l'estrade et les chaises disposées en demi-cercle -, chacun des doctorants a synthétisé l'état actuel de sa recherche en une vingtaine de minutes, portant l'accent sur les problèmes de méthode rencontrés et sur les (premiers) résultats d'ores et déjà obtenus. Une large place a ainsi pu être ménagée pour débattre avec un public fourni, composé à la fois d'universitaires et d'étudiants. Les communications étaient rassemblées en quatre blocs thématiques: histoire de l'art, minorités, histoire politique, société et économie. Les deux premières intervenantes, M. Miquel et C. Sanjust Latorre, ont analysé respectivement la situation économique et sociale des peintres dans le royaume de Valence (1370-1430) et l'architecture du monastère de Pedralbes à Barcelone. L'adoption d'une perspective d'histoire sociale de l'art (conditions de production, organisation de l'atelier, demande du marché), puis l'utilisation pour l'étude d'un monastère de clarisses des notions de paysage visuel et de variabilité des bâtiments ont donné un aperçu de l'ouverture croissante en Espagne de l'histoire de l'art vers des disciplines voisines.

Pour sa part, s'inscrivant dans une tradition historiographique très fournie - on pense ici au célèbre ouvrage de D. Niremberg, Violence et minorités au Moyen Âge - C. Cailleaux a montré le parti que l'on pouvait tirer d'une mise en regard de la situation des juifs et des musulmans dans la Couronne. De manière complémentaire, $\mathrm{Cl}$. Soussen a exploité la notion de «rencontre religieuse», démontrant à l'aide du cas exemplaire du prédicateur Ramon Martí comment l'on assistait dans la seconde moitié du XIII siècle à l'émergence d'un nouveau discours antijuif, fondé sur une connaissance profonde des textes hébraïques. La politique a ouvert l'après-midi.

L'auteur de ces lignes a examiné la diplomatie de Jacques II (12911327), remarquable à la fois par les modifications des pratiques institutionnelles qu'elle engendre et par le regard qu'elle permet de poser sur le pouvoir effectif du roi dans la Couronne. La réflexion a été poursuivie par A. Beauchamp, qui a montré comment la lieutenance de l'infant Pierre d'Aragon (1354-1355) révélait les mécanismes de la délégation 
du pouvoir et, corrélativement, délimitait les prérogatives royales. Dans la dernière section, E. Redondo García a souligné l'importance des prélèvements fiscaux extraordinaires, notamment pour qui s'intéresse à la nature des justifications employées par le pouvoir. Enfin, C. Vela i Aulesa a exploité socialement les riches testaments des épiciers et des vendeurs de bougies de Barcelone, avant que D. Duran i Duelt ne clôture la journée par l'analyse de l'impact d'une institution, le consulat des Catalans, sur le développement économique de la Couronne en Méditerranée orientale.

Ce panorama hétéroclite et subjectif de la recherche reflète assez bien, à sa manière, la vitalité et le caractère éclaté des travaux en cours sur la couronne d'Aragon au Moyen Âge. Le seul courant historiographique commun semble être ici l'accent porté sur la période s'étendant $\mathrm{du} \mathrm{XIII}^{\mathrm{e}}$ au XV $\mathrm{XV}^{\mathrm{e}}$ siècle, essentiellement en raison des fabuleuses ressources des archives catalanes. Sinon, l'espace des territoires de la couronne d'Aragon fonctionne de plus en plus comme le laboratoire d'expérimentation des problématiques les plus diverses, et ce trait distinctif aurait sans doute été plus visible encore si des doctorants américains avaient pu participer à la rencontre. En somme, au-delà de son utilité érudite pratique (la fiscalité a éclairé la diplomatie et le commerce des icônes le marché de la peinture), cette journée a surtout mis en exergue le danger et la nécessité de faire une histoire lisible et articulée de manière problématique d'un ensemble composite et pourtant unitaire. L'exercice périlleux de la comparaison entre les différents territoires - de la Couronne ou d'ailleurs -, trop souvent utilisé comme simple recours rhétorique, est apparu aussi indispensable que difficile à mettre en pratique, en raison de l'absence fréquente de tradition(s) historiographique(s) solide(s). La recherche d'une unité se manifeste aussi de manière récurrente par le renouvellement des interrogations sur le pouvoir royal aragonais, dont bien des aspects demeurent à explorer, sans parler de l'Église, la grande absente de cette journée, et des travaux actuels sur la couronne d'Aragon.

En dépit de ses limites évidentes, cette rencontre a donc contribué à la fois à modifier modestement les conditions de travail des doctorants et à identifier un peu plus clairement les raisons pour lesquelles l'écriture de l'histoire de la couronne d'Aragon s'apparente parfois à un véritable casse-tête chinois. Une seule réelle conclusion s'impose: il reste encore beaucoup à faire. 